\title{
Genome-wide analysis of the Populus Hsp90 gene family reveals differential expression patterns, localization, and heat stress responses
}

Jin Zhang, Jianbo Li, Bobin Liu, Li Zhang, Jun Chen ${ }^{*}$ and Mengzhu Lu*

\begin{abstract}
Background: Members of the heat shock protein 90 (Hsp90) class of proteins are evolutionarily conserved molecular chaperones. They are involved in protein folding, assembly, stabilization, activation, and degradation in many normal cellular processes and under stress conditions. Unlike many other well-characterized molecular chaperones, Hsp90s play key roles in signal transduction, cell-cycle control, genomic silencing, and protein trafficking. However, no systematic analysis of genome organization, gene structure, and expression compendium has been performed in the Populus model tree genus to date.
\end{abstract}

Results: We performed a comprehensive analysis of the Populus Hsp90 gene family and identified 10 Populus Hsp90 genes, which were phylogenetically clustered into two major groups. Gene structure and motif composition are relatively conserved in each group. In Populus trichocarpa, we identified three paralogous pairs, among which the PtHsp90-5a/PtHsp90-5b paralogous pair might be created by duplication of a genome segment. Subcellular localization analysis shows that PtHsp90 members are localized in different subcellular compartments. PtHsp90-3 is localized both in the nucleus and in the cytoplasm, PtHsp90-5a and PtHsp90-5b are in chloroplasts, and PtHsp90-7 is in the endoplasmic reticulum (ER). Furthermore, microarray and semi-quantitative real-time RT-PCR analyses show that a number of Populus Hsp90 genes are differentially expressed upon exposure to various stresses.

Conclusions: The gene structure and motif composition of PtHsp90s are highly conserved among group members, suggesting that members of the same group may also have conserved functions. Microarray and RT-PCR analyses show that most PtHsp90s were induced by various stresses, including heat stress. Collectively, these observations lay the foundation for future efforts to unravel the biological roles of PtHsp90 genes.

Keywords: Expression analysis, Gene family, Gene structure, Hsp90, Phylogenetic analysis, Populus

\section{Background}

Plants are exposed to various environmental stresses. Primary stresses such as high light intensity, heat shock, drought, chilling, salinity, and chemical pollutants act simultaneously on plants, causing cell injury and producing secondary stresses such as osmotic and oxidative stresses [1]. Plants cannot avoid exposure to these factors, but adapted morphologically and physiologically by some mechanisms. Biosynthesis of many proteins called "stress proteins" is induced to protect cells from these harmful stimuli [2].

\footnotetext{
* Correspondence: chenjun@caf.ac.cn; lumz@caf.ac.cn

State Key Laboratory of Tree Genetics and Breeding, Research Institute of Forestry, Chinese Academy of Forestry, Beijing 100091, China
}

Heat shock proteins (Hsps) are responsible for protein folding, assembly, translocation, and degradation in many normal cellular processes. They stabilize proteins and membranes, and can assist in protein refolding under stress conditions. They also play a crucial role in protecting plants from stresses by reestablishing normal protein conformations and thus cellular homeostasis [1]. Plant Hsps are classified into five families according to their molecular size: Hsp100, Hsp90, Hsp70, Hsp60, and small Hsps (sHsps). They have been well characterized in a few model plants such as the tomato, Arabidopsis, and rice $[3,4]$.

Hsp90s are a class of chaperone proteins that are highly conserved in prokaryotes and all eukaryotes. They are the major species of molecular chaperones and require ATP

\section{Biomed Central}


for their functions [1]. Although Hsp90s are expressed in most organisms, their expression increases in response to stresses. Distinct from many other well-characterized molecular chaperones, Hsp90s display considerable specificity for their client proteins. Most of their known substrates are signal-transduction proteins such as steroid hormone receptors and signaling kinases [5]. Although the major function of Hsp90s is to assist protein folding, they play key roles in signal transduction, cell-cycle control, protein degradation, genomic silencing, and protein trafficking [5,6]. Expression of Hsp90 in Arabidopsis is developmentally regulated and is responsive to heat, cold, salinity, heavy metals, phytohormones, and light and dark transitions [4,7]. Tobacco NbHsp90-1 and Arabidopsis AtHsp90-2 confer pathogen resistance by reacting to resistance proteins ( $R$ proteins), which are signal receptors from the pathogen $[8,9]$. In addition, Hsp90s interact with the $26 \mathrm{~S}$ proteasome and play a key role in its ATP-dependent assembly and maintenance in budding yeast [10]. To fulfill their cellular roles, Hsp90s cooperate with other chaperones to form a multiprotein chaperone complex [11]. Moreover, Hsp90s also act as buffers to phenotypic changes and are portrayed as "capacitors for evolution" [12].

Hsp90s are encoded by multiple genes. They consist of conserved $\mathrm{N}$-terminal and $\mathrm{C}$-terminal domains that are joined by a charged linker region that varies in length. Genes encoding cytosol-, ER-, and plastid-localized Hsp90 proteins have been characterized in several plant species [4]. In the Arabidopsis genome, seven Hsp90 family members have been identified. Sequence analyses of Arabidopsis Hsp90 family genes have revealed two major subfamilies. AtHsp90-1-4 proteins containing the C-terminal pentapeptide MEEVD form the cytoplasmic subfamily; AtHsp90-5-7 form the other subfamily. AtHsp90-5 and AtHsp90-7 are localized in chloroplasts [13] and the endoplasmic reticulum (ER) [14], respectively. AtHsp90-6 is localized in mitochondria [15]. Overexpression of cytosolic AtHsp90-2, chloroplast-localized AtHsp90-5, and ER-localized AtHsp90-7 reduces tolerance to salt and drought stresses, but improves tolerance to high concentrations of $\mathrm{Ca}^{2+}[16]$. The induction of ABAresponsive genes is delayed by overexpression of cytosolic AtHsp90-2, but is hardly affected by overexpression of AtHsp90-5 and AtHsp90-7 under conditions of salt and drought stress, which implies that different cellular compartm

ent-localized Hsp90s in Arabidopsis might contribute to responses to abiotic stresses by different functional mechanisms, probably through ABA- or $\mathrm{Ca}^{2}$ ${ }^{+}$-dependent pathways [16].

The Populus genus comprises woody plants that are important to humans and animals. Completion of the $P$. trichocarpa genome sequence in 2006 rendered it a model species for research on trees [17], providing an opportunity to analyze and further understand Hsp90s. To determine the structure-function relationship of Hsp90s in the Populus genus, we performed detailed systematic analyses of genome organization, gene structure, and expression compendium. We report the comprehensive genomic identification and phylogenetic analysis of all 10 members of the $H s p 90$ gene family in the Populus genus, as well as their expression profiles in different tissues and their responses under heat stress. Our results provide a framework for further functional investigations of these genes.

\section{Results and discussion}

\section{Identification of the Hsp90 gene family in P. trichocarpa} and other plant species

To identify putative Populus Hsp90 genes, we first searched relevant databases using the corresponding Arabidopsis Hsp90 protein sequences as queries. Additional searches were performed based on keyword querying. After removing redundant sequences, we identified 10 candidate $H s p 90$ sequences in the genome of P. trichocarpa. All PtHsp90 candidates were analyzed using the Conserved Domain Database (CCD) [18,19] and Pfam (http://pfam.sanger.ac.uk/). It was previously reported that there are seven $H s p 90$ genes presented in Arabidopsis [4]. The number of Hsp90 genes in $P$. trichocarpa genome is in consistency with the ratio of 1.4-1.6 putative poplar homologs for each Arabidopsis gene according to comparative genomics studies [17]. This indicates that the higher number of Hsp90 members in poplar is due to the expansion of gene families during the genome duplication and the genomic evolution followed. The Hsp90 genes identified in $P$. trichocarpa encode proteins ranging from 698 to 823 amino acids (aa) in length, with predicted isoelectric points (pIs) ranging from 4.85 to 5.53 (Table 1). The polypeptides are also predicted to contain a Histidine kinase-like ATPases (HATPase_c) family motif and a Hsp90 family motif (Additional file 1). HATPase_c domain belongs to the ATP binding superfamily including diverse protein families such as DNA topoisomerase II, molecular chaperones Hsp90, DNA-mismatch-repair enzymes, phytochrome-like ATPases and histidine kinases [20]. Detailed information on the Hsp90 family genes in P. trichocarpa, Arabidopsis, and rice is given in Table 1 and Additional file 1.

To investigate the evolutionary relationships of Hsp90 proteins from different plants, we identified Hsp90 genes from seven other plant species, including the moss Physcomitrella patens, the monocotyledonous angiosperms Oryza sativa, Sorghum bicolor, and Brachypodium distachyon, the dicotyledonous angiosperms Arabidopsis thaliana, Vitis vinifera, and Medicago truncatula. All angiosperm genomes, as well as the moss genome, contain 
Table 1 Hsp90 genes families in Arabidopsis, Populus, and rice

\begin{tabular}{|c|c|c|c|c|c|}
\hline Gene name & Locus & Genomic position & Mol. Wt. (kDa), Length (aa), pl & Gene length, ORF, introns & POSRT predictions* \\
\hline AtHsp90-1 & At5g52640 & Chr5:21352542-21355147 (+) & $81.18,705,4.95$ & $2606,2118,3$ & $\mathrm{~N}: 6, \mathrm{C}: 5, \mathrm{Ch}: 2$ \\
\hline AtHsp90-2 & At5g56030 & Chr5:22686923-22689433 (+) & $80.06,699,4.95$ & $2511,2187,2$ & $C: 11, P: 2$ \\
\hline AtHsp90-3 & At5g56010 & Chr5:22681410-22683911 (+) & $80.05,699,4.95$ & $2502,2100,2$ & $C: 9, P: 2, N: 1, M: 1$ \\
\hline AtHsp90-4 & At5g56000 & Chr5:22677602-22680067 (-) & $80.14,699,4.96$ & $2466,2100,2$ & $C: 9, P: 4$ \\
\hline AtHsp90-5 & At2g04030 & Chr2:1281983-1285909 (+) & $88.66,780,4.93$ & $3927,2343,18$ & Ch: $12, \mathrm{~N}: 1$ \\
\hline AtHsp90-6 & At3g07770 & Chr3:2479611-2483970 (+) & $90.57,799,5.26$ & $4360,2400,19$ & M: 9.5,Ch_M: 6.5, Ch: 2.5 \\
\hline AtHsp90-7 & At4g24190 & Chr4:12551902-12555851 (-) & $94.2,823,4.94$ & $3950,2472,14$ & E.R.: 7, V: 4, N: 1 \\
\hline PtHsp90-1a & Potri.004G073600 & Chr04: 6143799 - 6146941(-) & $80.72,703,5$ & $2566,2112,3$ & C: 7, N: 3, P: 2, M: 1 \\
\hline PtHsp90-16 & Potri.017G146600 & Chr17: 15369800 - 15373207(+) & $80.75,703,4.98$ & $2696,2112,3$ & C: $8, N: 2, P: 2, M: 1$ \\
\hline PtHsp90-2 & Potri.001G466000 & Chr01: 49936276 - 49939430(+) & $80.03,699,4.95$ & $2767,2100,2$ & C: 6, P: 2, E.R.: 2, Ch: 1, N: 1, M: 1 \\
\hline PtHsp90-3 & Potri.016G003400 & Chr16: $167702-171110(+)$ & $79.95,698,4.94$ & $2906,2097,3$ & $\mathrm{~N}: 7, \mathrm{C}: 5, \mathrm{Ch}: 2$ \\
\hline PtHsp90-4a & Potri.001G286700 & Chr01: 29295547 - 29298729(+) & $80.86,706,4.88$ & $2734,2121,2$ & C: 7, N: 2, P: 2, Ch: 1, M: 1 \\
\hline PtHsp90-4b & Potri.006G002800 & Chr06: 219117 - 222439(+) & $80.01,699,4.91$ & $2734,2100,2$ & $\mathrm{~N}: 4, \mathrm{C}: 4, \mathrm{P}: 3, \mathrm{Ch}: 1, \mathrm{~V}: 1$ \\
\hline PtHsp90-5a & Potri.008G112700 & Chr08: 7190800 - 7197203(+) & $90.16,791,4.92$ & $5884,2376,17$ & Ch: 10, N: 2, P: 1 \\
\hline PtHsp90-5b & Potri.010G136800 & Chr10: 15004039 - 15010595(-) & $90.15,793,4.96$ & $5723,2382,17$ & Ch: 13 \\
\hline PtHsp90-6 & Potri.014G164900 & Chr14: 13043761 - 13049852(-) & $82.95,723,5.53$ & $4850,2172,13$ & M: 7.5, Ch_M: 7, Ch: 5.5 \\
\hline PtHsp90-7 & Potri.005G241100 & Chr05: 24712685 - 24717968(-) & $94.05,823,4.85$ & $4926,2472,14$ & E.R.: 5, V: 5, P: 2, Ch: 1 \\
\hline OsHsp90-1 & LOC_Os04g01740 & Chr4: 483241 - $486065(+)$ & $80.25,703,5.04$ & $2353,2112,2$ & C: 11, Ch: $1, P: 1$ \\
\hline OsHsp90-2 & LOC_Os08g39140 & Chr8: 24719086 - $24723553(-)$ & $80.19,699,4.99$ & $4002,2100,2$ & C: 8, P: 2, Ch: 1, N: 1, M: 1 \\
\hline OsHsp90-3 & LOC_Os09g30412 & Chr9: $18514572-18518316(-)$ & $80.2,699,4.97$ & $3232,2100,2$ & N: 7, C: 4, Ch: 2 \\
\hline OsHsp90-4 & LOC_Os09g30418 & Chr9: 18535746 - $18541109(-)$ & $94.2,830,5.15$ & $5364,2493,3$ & $\mathrm{~N}: 8, \mathrm{C}: 3, \mathrm{Ch}: 1, \mathrm{P}: 1$ \\
\hline OsHsp90-5a & LOC_Os08g38086 & Chr8: 24124838 - $24129488(-)$ & $85.85,761,4.97$ & $4285,2286,19$ & Ch: 12.5, Ch_M: 7 \\
\hline OsHsp90-5b & LOC_Os09g29840 & Chr9: 18150618 - $18155512(-)$ & $89.22,791,5.03$ & $4359,2376,18$ & Ch: 12.5, Ch_M: 7 \\
\hline OsHsp90-6 & LOC_Os12g32986 & Chr12: 19921576 - 19927766 (-) & $91.48,811,5.21$ & $5680,2436,19$ & Ch: 12, M: 1 \\
\hline OsHsp90-7 & LOC_Os06g50300 & Chr6: 30444411 - $30450497(-)$ & $93.04,812,4.89$ & $5669,2439,14$ & E.R.: 5, V: 5, P: 2, Ch: 1 \\
\hline
\end{tabular}

Gene loci are obtained from the Phytozome website (http://www.phytozome.net). A complete list of the coding sequences (CDS), deduced amino acid sequences and genomic DNA sequences is available in Additional file 2. *PSORT predictions: P (plasma membrane), V (vacuolar membrane), C (cytosol), Ch (chloroplast), N (nuclear), E.R. (endoplasmic reticulum), $\mathrm{M}$ (mitochondrion). The numbers indicate the number of nearest neighbors to the query which localize to each site. 
Hsp90 genes. The number of Hsp90s identified is seven in A. thaliana, ten in P. trichocarpa, five in $V$. vinifera, five in M. truncatula, eight in O. sativa, seven in S. bicolor, eight in $B$. distachyon, and ten in $P$. patens. Additional file 3 provides a complete list of all $H s p 90$ genes identified in the present study.

\section{Phylogenetic analyses of the $\mathrm{Hsp} 90$ gene family}

To examine the phylogenetic relationships among the Hsp90 genes in P. trichocarpa and other plant species, we first generated a maximum likelihood phylogenetic tree by aligning full-length Hsp90 protein sequences from eight different plant species using PhyML. All of the sequences are classified into two major groups (group I and II), each of which is further divided into two subgroups (subgroup Ia, Ib, IIa and IIb) (Figure 1). The distribution of Hsp90 members in different species varies, and subgroups Ib and IIa are the largest two subgroups. There are two subgroup Ia members in $P$. trichocarpa, but none in moss and only one in the other species examined (Table 2). There are also more group Ib Hsp90 members in moss than these in the other species analyzed.

Next, we constructed a phylogenetic tree of the Hsp90 protein sequences from Populus, Arabidopsis, and rice using the neighbor-joining (NJ) method (Figure 2A). The tree topologies produced by two algorithms are largely comparable, with only minor differences at interior branches (Figure 2A). Distance and percentage of identity among Populus, Arabidopsis, and rice Hsp90 proteins are given in Additional file 4. Phylogenetic analysis shows that there is high similarity among the cytosolic members and less similarity among the organelle-type members. In addition, both trees show that the most recent duplicated pairs (Hsp90-1a/Hsp90-1b, Hsp90-4a/Hsp90-4b and Hsp90-5a/Hsp90-5b) exhibit high similarity, which indicates that they evolved slowly in sequence and structure, and may still keep their function.

It is more accurate to reflect an evolutionary relationship by using conserved domain sequences [18]. Therefore we also constructed the phylogenetic tree with the conserved Hsp90 motif sequences from Populus, Arabidopsis, and rice using the maximum likelihood method with 1000 bootstrap replicates (Additional file 6). The resulted phylogenetic tree is consistent with the one generated based on the full length protein sequences.

\section{Gene structure and conserved motifs of $\mathrm{Hsp} 90$ genes in Populus, Arabidopsis, and rice}

To further investigate the structural diversity of $H s p 90$ genes in Populus, Arabidopsis, and rice, we first constructed a separate phylogenetic tree using the full-length Hsp90 protein sequences from these three species. The
Hsp90 proteins are classified into two groups as described above (Figure 2A). Then we analyzed the exon/intron organization in the coding sequence of each $H s p 90$ gene (Figure 2B). In general, the positions of some spliceosomal introns are conserved in orthologous genes. In many cases, conservation of exon/intron organization or gene structure in paralogous genes is high and sufficient to reveal the evolutionary relationship between introns [21]. In the present study, Hsp 90 gene family members within the same group shared similar gene structures in terms of intron number or exon length (Figure 2B). Hsp90 group I comprises the cytosolic Hsp90s whose members have two or three introns, while group II comprises organelle-type Hsp90s, which have 13-19 introns (Figure 2 and Table 1). The gene structure difference between group I and group II Hsp90 might associate with their functions in different biological processes in subcellular compartments. We also investigated intron phases with respect to codons. The intron phases are remarkably well conserved among group members, while the intron arrangements and intron phases are strikingly distinct between groups (Figure 2). This may lend support to the results of phylogenetic and genome duplication analyses. We further examined the exon/intron organization of paralogous pairs of $H s p 90$ genes to explore traceable intron gain or loss within these genes. Three paralogous pairs in Populus (PtHsp90-1a/1b, PtHsp 90-4a/4b, and PtHsp90-5a/5b) show conserved exon/intron structures in terms of intron number or gene length, while OsHsp90-5a shows a single intron gain event during the structural evolution of the OsHsp90$5 a / 5 b$ paralogous pair. Interestingly, OsHsp90-4 has an additional $\mathrm{C}$-terminal exon compared to other members of the group I Hsp90.

Next, we predicted the major domains of these proteins in all three species using Pfam and CDD [18]. All of the proteins contain a HATPase_c superfamily domain and a Hsp90 family domain (Additional file 1). Although the tools we used are suitable for defining the presence or absence of recognizable domains, they are unable to recognize smaller individual motifs and more divergent patterns. Thus, the program MEME was used to further study the diversification of these proteins [22]. Twenty distinct motifs were identified (Figure 2C). Details of the 20 motifs are presented in Additional file 5. Most of the closely related members have common motif composition, suggesting possible functional similarity among these Hsp90 proteins (Figure 2C). Motif 2 and 6 (corresponding to the HATPase_c superfamily domain at the $\mathrm{N}$-terminus) are found in all Hsp90 proteins from the species we examined. It has reported that both ATP binding and hydrolysis are required for Hsp90 function in vivo [23,24]. Noticeably, motif 20 (representing the LEA_6 subdomain) is only found in OsHsp90-4. This 


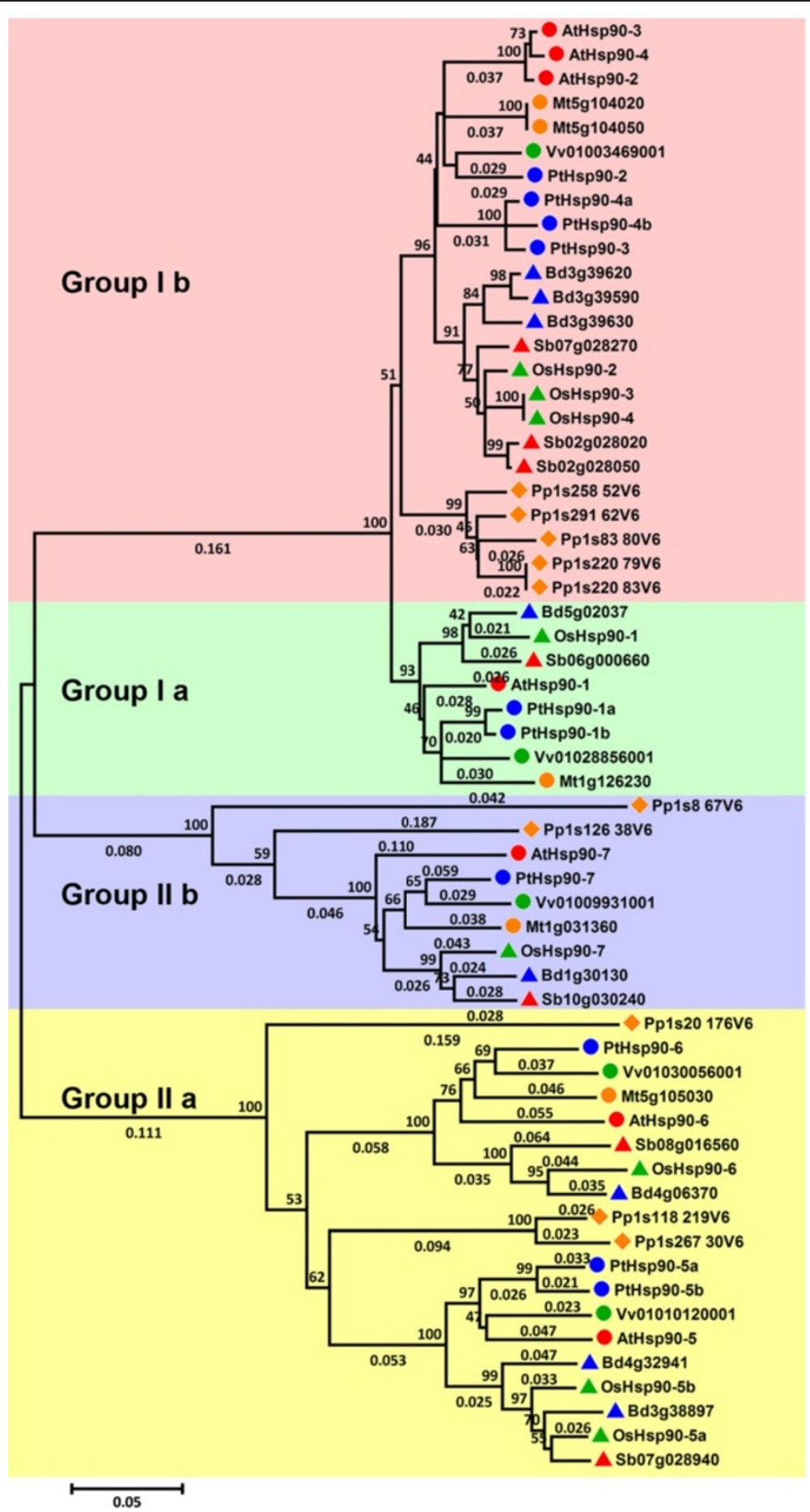

Figure 1 Phylogenetic relationships of Hsp90 family members from eight plant species. Multiple alignment of Hsp90 proteins from A. thaliana (At), P. trichocarpa (Pt), O. sativa (Os), M. truncatula (Mt), S. bicolor, (Sb), B. distachyon (Bd), V. vinifera (Vv), and P. patens (Pp) was performed using Clustal X2.1, and a phylogenetic tree was constructed using full-length protein sequences by the maximum likelihood method using PhyML. Bootstrap support values are shown on selected branches.

additional LEA_6 subdomain might explain the specific ability of OsHsp90-4 to acclimatize to various stresses.
Chromosomal location and gene duplication of Hsp90 genes in Populus, Arabidopsis, and rice

Chromosomal mapping of the gene loci shows that the 10 PtHsp90 genes are distributed unevenly among nine 
Table 2 Numbers of Hsp90s within each plant species

\begin{tabular}{lllll}
\hline & la & lb & Ila & Ilb \\
\hline A. thaliana & 1 & 3 & 2 & 1 \\
O. sativa & 1 & 3 & 3 & 1 \\
P. trichocarpa & 2 & 4 & 3 & 1 \\
V. vinifera & 1 & 1 & 2 & 1 \\
M. truncatula & 1 & 2 & 1 & 1 \\
S. bicolor & 1 & 3 & 2 & 1 \\
B. distachyon & 1 & 3 & 3 & 1 \\
P. patens & 0 & 5 & 3 & 2 \\
\hline
\end{tabular}

chromosomes (Additional file 7). Two PtHsp90 genes are localized on chromosome I, and one is localized on each of chromosome IV, V, VI, VIII, X, XIV, XVI, and XVII. Gene duplication events are thought to occur frequently in organismal evolution $[25,26]$. Previous studies report that the Populus genome has experienced at least two genome-wide duplication events (eurosid and salicoid), followed by a series of chromosomal reorganizations involving reciprocal tandem/terminal fusions and translocations [17]. To investigate the possible relationship between $H s p 90$ genes and segmental chromosome duplication, we also compared the locations of Hsp90 genes in duplicated chromosomal blocks that were previously identified in Populus, Arabidopsis, and rice $[17,27,28]$. Their distributions are shown in Additional file 7 (Populus), Additional file 8 (Arabidopsis), and Additional file 9 (rice). The results suggest that segmental duplication and transposition events are not the major factors that led to the expansion of the Populus $H s p 90$ gene family. It may be that dynamic changes occurred following segmental duplication and led to the loss of many of the duplicated Hsp 90 genes.

A search for duplicated genes using the Plant Genome Duplication Database (PGDD; http://chibba.agtec.uga. edu/duplication/) revealed the existence of three gene pairs (PtHsp90-1a/PtHsp90-1b, PtHsp90-3/PtHsp90-4b, and PtHsp90-5a/PtHsp90-5b) in P. trichocarpa (Additional file 10A) and two pairs (OsHsp90-2/OsHsp90-3 and OsHsp90$5 a / O s H s p 90-5 b$ ) in O. sativa (Additional file 10B). Interestingly, PtHsp $90-4 a$ was not assigned as a duplicated gene with PtHsp 90-3 and PtHsp 90-4b, indicating that PtHsp90$4 a$ had experienced intensive recombination events after the recent duplication with $P t H s p 90-4 b$, which led to the great divergence in its adjacent regions. Of the three

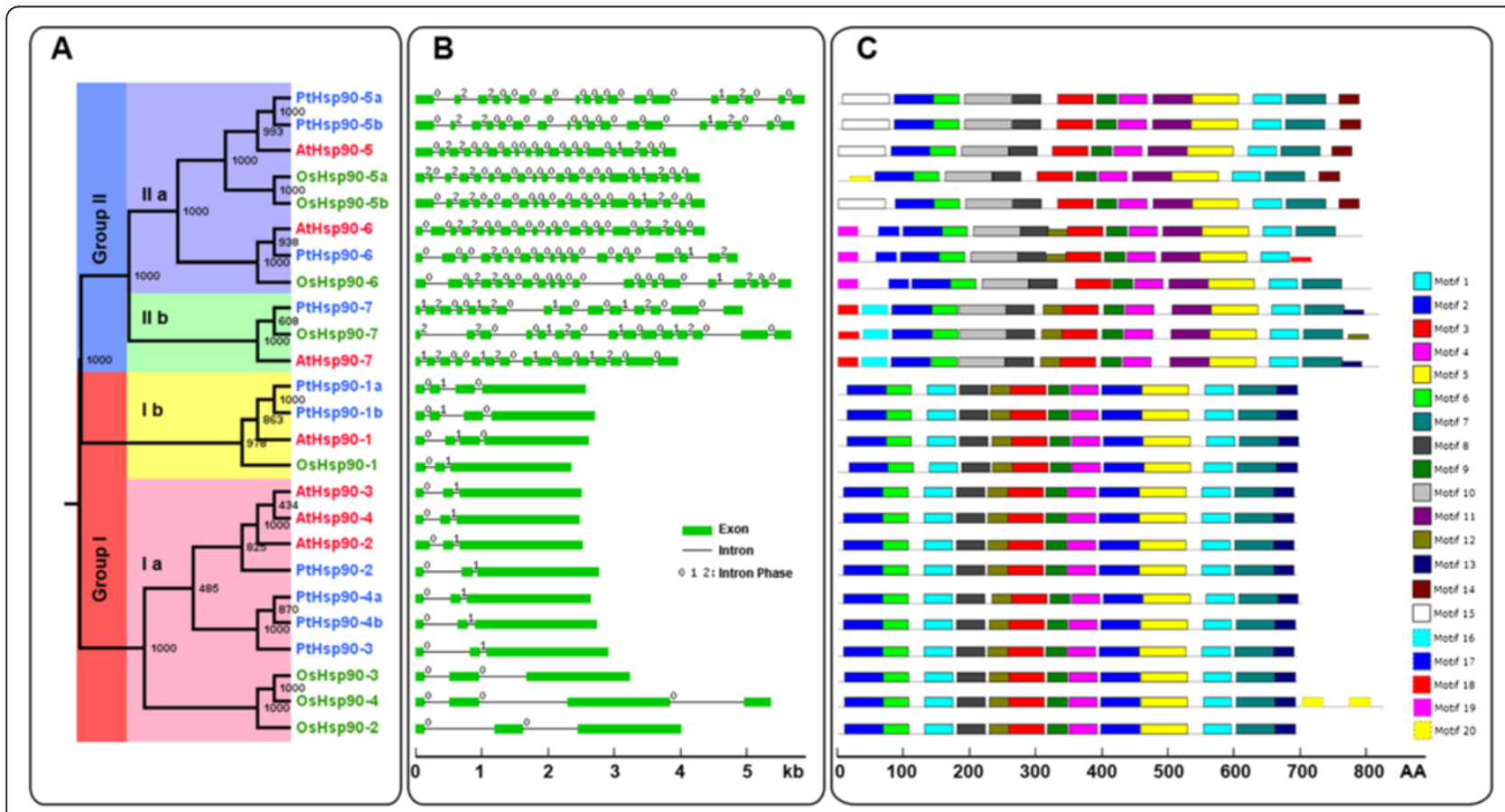

Figure 2 Phylogenetic relationships, gene structures, and motif composition of $\mathrm{Hsp} 90$ genes in $\mathrm{A}$. thaliana (At), P. trichocarpa (Pt), and O. sativa (Os). A. A multiple alignment of full-length Hsp90 protein sequences from three species was executed using Clustal X2.1 and a phylogenetic tree was constructed using MEGA 4.0 by the neighbor-joining ( $\mathrm{NJ}$ ) method with 1000 bootstrap replicates. Bootstrap support is indicated at each node. The two major groups are marked with different background colors. B. Exon/intron structures of the Hsp90 genes. Green boxes represent exons and black lines represent introns. The numbers indicate the splicing phases of the Hsp90 genes: 0, phase 0; 1, phase 1; and 2, phase 2. C. Schematic representation of conserved motifs (obtained using MEME) in Hsp90 proteins. Different motifs are represented by boxes of different colors. Details of the individual motifs are shown in Additional file 5. 
Hsp90 pairs in Populus that we examined, only one pair, PtHsp90-5a/PtHsp90-5b, remained in a conserved position in segmental duplicated blocks (Additional file 7), suggesting that only this paralogous pair survived during the evolutionary process after chromosome duplication event.

\section{Subcellular localization of Populus Hsp90 proteins}

In silico analyses using the protein subcellular localization prediction software WoLF PSORT (http://wolfpsort.org) enabled us to predict the likely protein localization of each of the different candidate Hsp90s in Populus. PtHsp90-3 is predicted to be localized in the nucleus or in the cytosol with high reliability, while PtHsp90-5a and PtHsp90-5b are predicted to be localized in chloroplasts, PtHsp90-6 is predicted to be localized in mitochondria, and PtHsp90-7 is predicted to be localized in the ER. For the other PtHsp90 proteins, the cytosol is predicted to be their most likely location (Table 1). To confirm their predicted localizations, some of these proteins were transiently expressed in tobacco leaf epidermal cells as fusions with the $\mathrm{N}$ terminus of YFP. Four Hsp90 proteins were successfully expressed as fluorescent protein fusions (PtHsp90-3-YFP, PtHsp90-5a-YFP, PtHsp90-5b-YFP, and YFP-PtHsp90-7). Based on sequence analysis, PtHsp90-1a, PtHsp90-1b, PtHsp90-2, PtHsp90-3, PtHsp90-4a, and PtHsp90-4b contain the C-terminal pentapeptide MEEVD (Additional file 2), which is characteristic of cytoplasmic Hsp90 proteins both in plants and in animals. In Arabidopsis, it was confirmed that two cytoplasmic Hsp90s (AtHsp90-1 and AtHsp90-3) are localized both in the nucleus and in the cytoplasm [29]. As shown in Figure 3A, the fluorescent signal of PtHsp90-3-YFP is also detected both in the nucleus and in the cytoplasm. This is consistent with the subcellular localizations of cytoplasmic Arabidopsis Hsp90 proteins $[4,15]$. Using the autofluorescence of chlorophyll as a marker, we found that the fluorescent signals of both PtHsp90-5a-YFP and PtHsp90-5b-YFP are well colocalized with red chlorophyll autofluorescence (Figure 3B and $3 \mathrm{C}$ ). A transit peptide for the import into mitochondria was identified in the N-terminal region of PtHsp90-6, but the intercellular localization of PtHsp90-6 remains to be confirmed experimentally. The PtHsp90-7 protein sequence contains a C-terminal KDEL ER-retention motif (Additional file 2). When YFP-PtHsp90-7 is co-expressed with the well-characterized luminal ER marker GFPHDEL [30], it is co-localized with GFP-HDEL (Figure 3D), which confirms its ER localization. These results suggest that the localization of Hsp90s in the same subgroup is relatively conserved among different species. The conserved organelle localization of Hsp90 implies that they might play roles in organelle-specific development or stress response. It has been suggested that mutation of the chloroplast-localized AtHsp90-5 causes altered response to red light, chlorate resistance and constitutively delayed chloroplast development in the $\mathrm{cr} 88 \mathrm{mu}$ tant $[13,31,32]$. In animals, a mitochondrial-localized Hsp90 appeared to have a critical role in cell cycle progression, cellular differentiation, and apoptosis [33,34]. In tobacco, mitochondrial-localized Hsp90 was involved in the $N$ gene-dependent cell death by affecting downstream MAPK cascade function [35]. Mutation of the ERlocalized AtHsp90-7 produced floral and shoot meristem phenotypes in the shepherd mutant that closely resemble that of the three clavata (clv) mutants in Arabidopsis $[14,36,37]$. The conserved subcellular localization of Hsp90s might provide clues for their specific cellular functions.

\section{Differential expression patterns of $\mathrm{Hsp} 90$ genes in Populus}

The expression patterns of genes can provide useful clues for the functions of these genes. To verify the expression profiles of Populus Hsp90 genes, the RNA-seq data of different Populus vegetative tissues (unpublished data) were used to analyze the expression of PtHsp 90 genes. PtHsp 90-5a and PtHsp90-5b are mainly expressed in the young leaves (YL) and mature leaves (ML) (Figure 4A), which is consistent with their localization in chloroplasts (Figures 3B and 4C). The expression of PtHsp90-5a in the young leaves is stronger than that in the mature leaves, suggesting PtHsp90-5a may play roles in young leaf development. The other PtHsp90 genes are mainly expressed in stems including primary stem (PS) or secondary stem (SS). PtHsp90-1b is highly expressed in secondary stem, while PtHsp90-1a, PtHsp90-6 and PtHsp90-7 are mainly expressed in primary stem. These results imply that these PtHsp90s might be involved in different stages of stem development. The transcription levels of PtHsp90-2, PtHsp90-3, PtHsp90-4a and PtHsp90-4b are higher than these of the other PtHsp90 members. PtHsp90-4a and PtHsp90-4b are ubiquitously highly expressed in almost all detected tissues (Additional file 11). In order to verify the expression profiles of PtHsp 90 genes obtained by RNAseq, qRT-PCR analysis of seven selected PtHsp 90 genes was performed on three different tissues (Figure 4B). The average expression of each gene was calculated relatively to the value of the first replication of roots \pm standard error $(\mathrm{SE})(\mathrm{n} \geq 3)$. The gene expression pattern detected by qRT-PCR is generally consistent with the RNA-seq results. The different expression patterns of PtHsp90s in different tissues imply that PtHsp90 members may be involved in different biological processes.

\section{Differential stress responses of Hsp90 genes in Populus}

In order to reveal the responses of Populus Hsp 90 genes to abiotic stresses, we analyzed the expression profiles of PtHsp90s under abiotic stresses such as heat, low nitrogen levels, mechanical wounding, drought, and methyl 

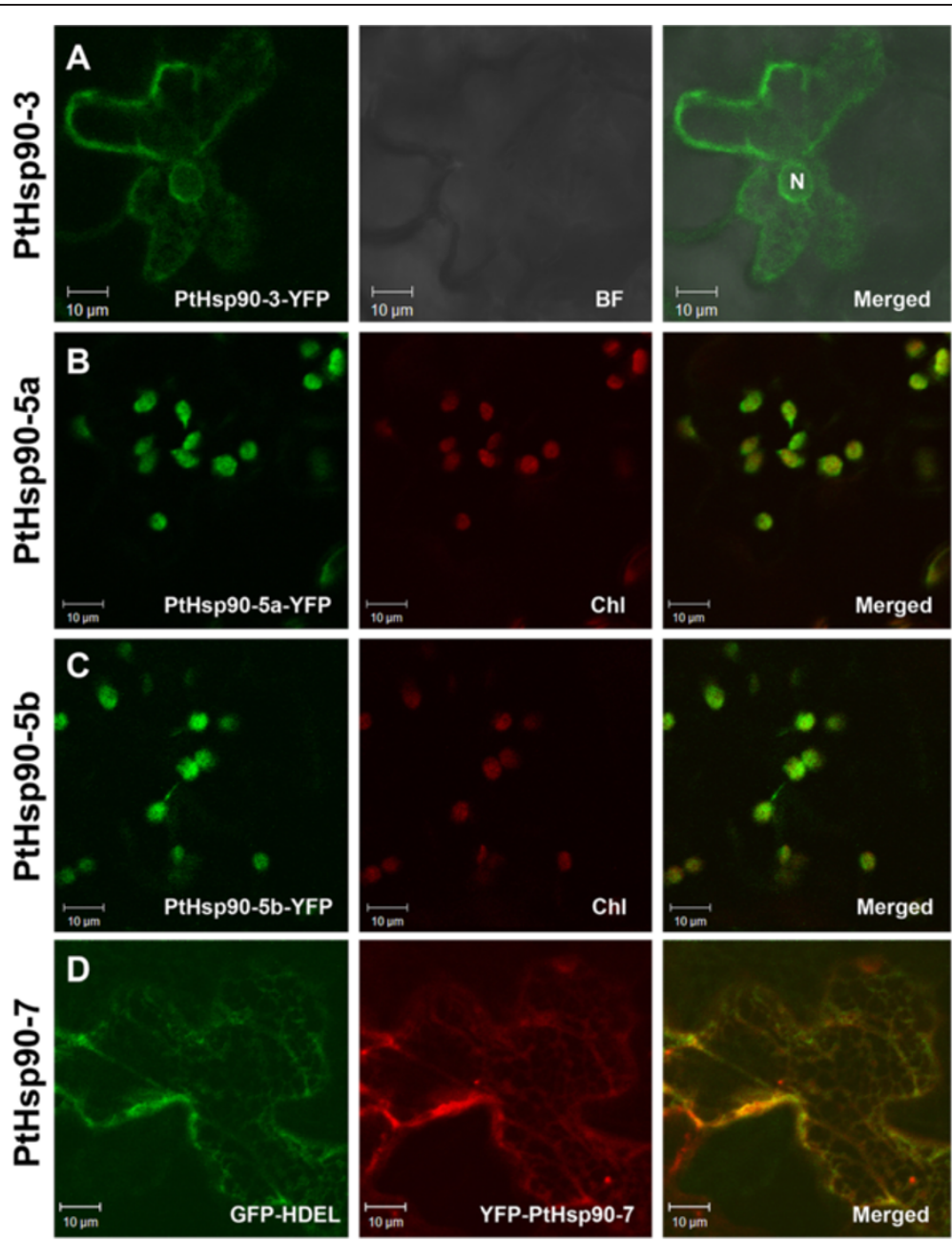

Figure 3 Subcellular localization of PtHsp90 proteins. A. Confocal image of an epidermal leaf cell expressing PtHsp90-3-YFP. B-C. Confocal images of epidermal leaf cells expressing PtHsp90-5a-YFP and PtHsp90-5b-YFP. The red channel shows autofluorescence of chlorophyll in photosynthetic tissues. D. Confocal images of epidermal leaf cells co-expressing YFP-PtHsp90-7 (red channel) and GFP-HDEL (green channel). Scale bar $=10 \mu \mathrm{m}$.

jasmonate (MeJ) treatment. Affymetrix microarray data (series accession numbers GSE26199, GSE16786 and GSE17230 in the Gene Expression Omnibus [GEO]) [38] were used to analyze the global expression profiles of Populus Hsp90 genes. Previous study divided the physiological condition into four states according to Populus photosynthetic activity from $22^{\circ} \mathrm{C}$ to $42^{\circ} \mathrm{C}$ : baseline $\left(22^{\circ} \mathrm{C}\right.$, the growth temperature), optimum $\left(31.75^{\circ} \mathrm{C}\right.$, temperature producing the maximum net $\mathrm{CO}_{2}$ assimilation rate), $20 \%$ inhibition of optimum $\left(38.4^{\circ} \mathrm{C}\right)$ and $30 \%$ inhibition of optimum $\left(40.5^{\circ} \mathrm{C}\right)$ [39]. Most PtHsp 90 genes are upregulated under heat stress. The expression of PtHsp90-1a and PtHsp90-1b is highly induced immediately when temperature increases to optimum. PtHsp 90-5a and PtHsp90-6 are highly induced when the photosynthesis is inhibited by $30 \%$ under heat stress (Figure 5A). In PtHsp 90 group I, PtHsp90-1a, PtHsp90-
$1 b$, and PtHsp90-3 in both the Soligo and Carpacio genotypes are upregulated under almost all drought stresses tested, including the early response (EAR) to drought at $36 \mathrm{~h}$, and the long-term (10 days) responses to mild stress (LMI) and moderate stress (LMO) (Figure 5C). Nitrogen deficiency stress causes different responses among Hsp 90 genes. For instance, PtHsp $90-1 a$ and PtHsp90-1b are upregulated in 4-week-old young leaves (YL) and 8-week-old expanded leaves (EL) of genotype 1979 and genotype 3200; PtHsp90-5a and PtHsp90-5b are upregulated in 8-week-old expanded leaves (EL) of the same two genotypes. However, PtHsp90-3, PtHsp90-4a, and PtHsp90-6 are downregulated in 8-week-old expanded leaves in genotype 1979 and/or genotype 3200 (Figure 5B). In response to mechanical wounding stress, six genes (PtHsp90-1a, PtHsp90-1b, PtHsp90-3, PtHsp90-5b, PtHsp90-6, and PtHsp90-7) are significantly 

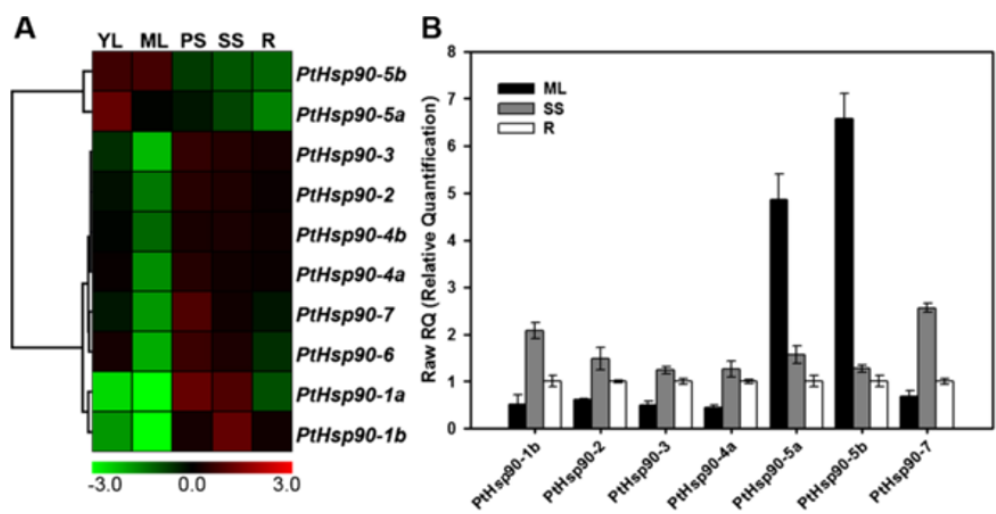

Figure 4 Expression analysis of PtHsp90 genes in different tissues. A. Heat map showing hierarchical clustering of PtHsp 90 genes in vegetative tissues (YL, young leaves; $M L$, mature leaves; $P S$, primary stem; SS, secondary stem; R, roots). The data was obtained from our unpublished RNA-seq data. The expression level of genes was determined based on the value of RPKM (reads per kilobase of exon region in a gene per million mapped reads). Details of the RPKM are shown in Additional file 11. Color scale represents log2 expression values. B. Expression of seven selected PtHsp90s was quantified by quantitative reverse-transcription polymerase chain reaction (qRT-PCR) in vegetative tissues (ML, mature leaves; SS, secondary stem; R, roots). The average expression of each gene was calculated relatively to the first biological replicate of roots \pm standard error (SE) $(n \geq 3)$.

downregulated in young leaves and/or expanding leaves 1 week after wounding. In response to MeJ feeding in cell culture, only PtHsp90-1a and PtHsp90-1b are slightly downregulated (Figure 5B).

The responses of PtHsp 90 genes to heat stress were analyzed experimentally. Heat-stress treatment comprising pretreatment for $3 \mathrm{~h}$ at $37^{\circ} \mathrm{C}$ and subsequent treatment at $45^{\circ} \mathrm{C}$ for $3 \mathrm{~h}$, with a 2 -h recovery interval, was performed. Most genes are induced by heat stress (Figure 6, Additional file 12). We classified the PtHsp90s into four classes according to their expression profiles under heat stress. Class I genes are induced immediately by both $37^{\circ} \mathrm{C}$ pretreatment and $45^{\circ} \mathrm{C}$ treatment (PtHsp $90 \mathrm{~s}$ in this class are positively regulated under both $37^{\circ} \mathrm{C}$ pretreatment and subsequent treatment at $45^{\circ} \mathrm{C}$ ) (Figure 6B). Notably, PtHsp $90-1 \mathrm{a}$ is induced $30 \mathrm{~min}$ after $37^{\circ} \mathrm{C}$

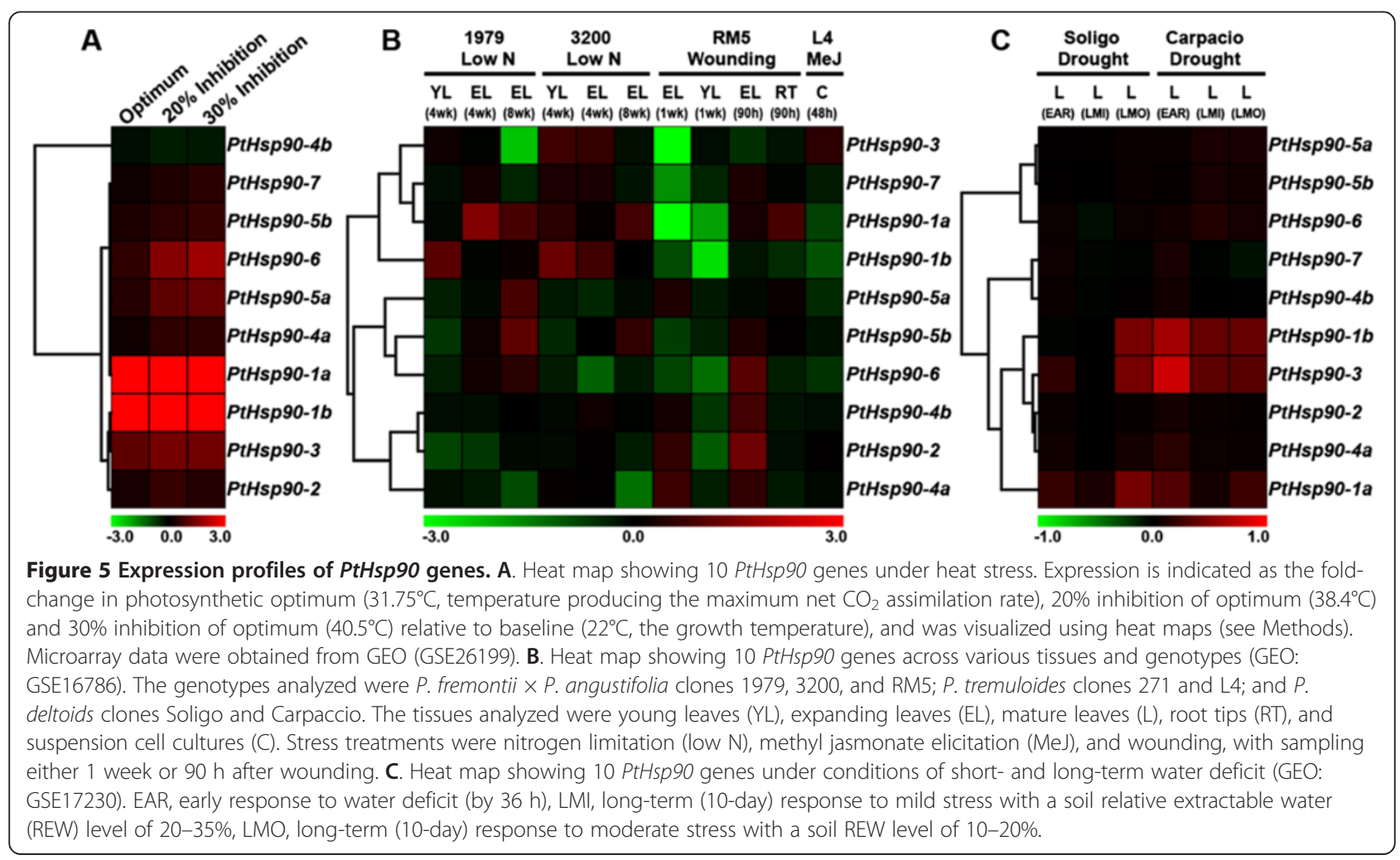




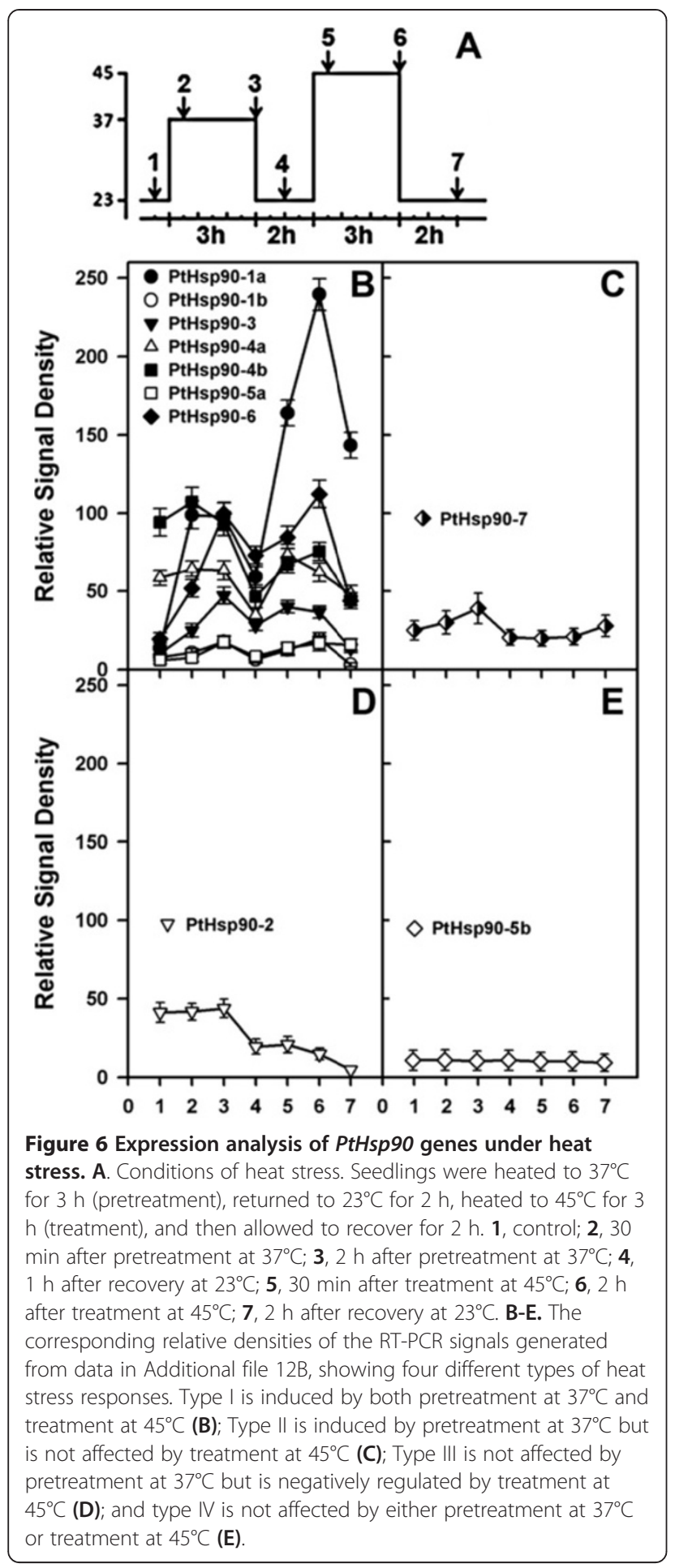

pretreatment and significantly induced $3 \mathrm{~h}$ after $45^{\circ} \mathrm{C}$ treatment in leaves. Class II genes are induced by $37^{\circ} \mathrm{C}$ pretreatment but not affected by $45^{\circ} \mathrm{C}$ treatment. PtHsp $90-7$ belongs to this class and its expression is induced by $37^{\circ} \mathrm{C}$ pretreatment. However, the expression of PtHsp90-7 is not affected by $45^{\circ} \mathrm{C}$ treatment following $2 \mathrm{~h}$ of recovery from $37^{\circ} \mathrm{C}$ pretreatment (Figure $6 \mathrm{C}$ ). Class III genes are not affected by $37^{\circ} \mathrm{C}$ pretreatment but are negatively regulated by $45^{\circ} \mathrm{C}$ treatment. PtHsp $90-2$ is not induced by $37^{\circ} \mathrm{C}$ pretreatment, and its mRNA abundance is reduced after recovery from $37^{\circ} \mathrm{C}$ pretreatment and subsequent $45^{\circ} \mathrm{C}$ treatment (Figure 6D). Class IV genes are not affected by either $37^{\circ} \mathrm{C}$ pretreatment or $45^{\circ} \mathrm{C}$ treatment significantly. The expression of PtHsp90-5b is still maintained in a low level in $37^{\circ} \mathrm{C}$ pretreatment and $45^{\circ} \mathrm{C}$ treatment (Figure 6E).

To verify the expression profiles of PtHsp90 genes in response to heat stress, qRT-PCR analysis was performed for four selected PtHsp90 genes under heat stress (Additional file 12C-F). Notably, PtHsp90-3, PtHsp90-4a and PtHsp 90-5a are induced $3 \mathrm{~h}$ after $37^{\circ} \mathrm{C}$ pretreatment and significantly induced $3 \mathrm{~h}$ after $45^{\circ} \mathrm{C}$ treatment in leaves (Additional file 12C-E). The expression of PtHsp90-5b is also induced by heat stress, but the induction is not that dramatic compared with that of the other PtHsp 90 genes in both $37^{\circ} \mathrm{C}$ pretreatment (2-fold) and $45^{\circ} \mathrm{C}$ treatment (3fold) (Additional file 12F). In addition, we found that the paralogous pair PtHsp90-5a/PtHsp90-5b shared the same expression profile in different tissues but were different under wounding and heat stresses (Figures 5 and 6). We then analyzed the promotors (2000 bp upstream of the start codon) of PtHsp90-5a and PtHsp90-5b using PlantCARE [40]. The sequence of the promotors share a low sequence identity (43.3\%) and two heat shock elements (HSE) exist in the promotor of PtHsp90-5a while none in PtHsp-5b (data not shown), which may contribute to the different expression pattern of the two genes. These results suggest different response mechanisms of PtHsp 90 members may exist under heat stress, and provide significant insights into their functions.

\section{Conclusions}

We performed a comprehensive analysis of the Populus Hsp90 gene family covering phylogeny, chromosomal location, gene structure, subcellular localization, expression profiling, and heat stress responses. A total of 10 full-length Hsp90 genes were identified in the Populus genome, all of which are clustered into two distinct groups. Exon/intron structure and motif compositions are found to be relatively conserved in each subgroup. The Populus genome contains three paralogous Hsp 90 gene pairs, but only PtHsp90-5a/PtHsp90-5b is located in conserved positions in duplicated blocks, suggesting that it may be derived from a segmental duplication event during evolution. Furthermore, subcellular localization analysis revealed that PtHsp90 members are localized in different organelles. In addition, comparative expression profile analysis of Populus Hsp90s revealed that Hsp90s may play various conserved roles in different biological processes in plants. Although the functions of PtHsp90s remain largely unknown and many 
experiments are needed to determine their precise functions, our phylogenetic and expression analyses of the Populus Hsp90 gene family establishes a solid foundation for future comprehensive functional analyses of PtHsp90s.

\section{Methods}

Database searching and sequence retrieval

To identify potential members of the Populus Hsp90 gene family, we performed multiple database searches. Published Arabidopsis Hsp90 gene sequences [4] were retrieved and used as queries in BLAST searches against the Poplar Genome Database (http://www.phytozome. net/poplar.php, release 3.0). BLAST searches were also performed against the poplar genomes at the National Center for Biotechnology Information (NCBI, http:// www.ncbi.nlm.nih.gov) and Phytozome (http://www. phytozome.net). Rice $H s p 90$ gene sequences were downloaded from the Rice Genome Annotation Project Database (http://rice.plantbiology.msu.edu/, release 7). Sequences of M. truncatula, S. bicolor, B. distachyon, $V$. vinifera, and $P$. patens were downloaded from Phytozome (http://www.phytozome.net). Local BLAST searches were performed using Arabidopsis Hsp90 protein sequences as queries to identify Hsp90 sequences in these plant species. All of the sequences were manually analyzed to confirm the presence of HATPase and Hsp90 domains using InterProScan (http://www.ebi.ac.uk/Tools/pfa/iprscan/).

WoLF PSORT (http://wolfpsort.org) was used to predict protein subcellular localization. The $\mathrm{pI}$ and molecular weight were estimated using the Compute $\mathrm{pI} / \mathrm{Mw}$ tool from ExPASy (http://web.expasy.org/compute_pi).

Functional motifs or domains of PtHsp90 sequences were analyzed using the PROSITE (http://prosite.expasy. org/) and Conserved Domain databases (http://www.ncbi. nlm.nih.gov/Structure).

\section{Phylogenetic analyses}

Multiple sequence alignment of the full-length protein sequences was performed using ClustalX2 (version 2.1) [41]. A maximum likelihood (ML) phylogenetic tree was constructed using PhyML (v3.0) with the JTT amino acid substitution model, 1000 bootstrap replicates, estimated proportions of invariable sites, four rate categories, estimated gamma distribution parameters, and an optimized starting BIONJ tree [42,43].

\section{Chromosomal location and gene structure of the Hsp90 genes}

The chromosomal locations of the Hsp 90 genes were determined using the Populus genome browser (http://www. phytozome.net/poplar). Information on intron/exon structure was collected from the genome annotations of $P$. trichocarpa from NCBI and Phytozome.

\section{Gene structure analysis}

The exon and intron structures of individual Hsp90 genes were illustrated using Gene Structure Display Server (GSDS, http://gsds.cbi.pku.edu.cn/) [44] by aligning the cDNA sequences with the corresponding genomic DNA sequences from Phytozome.

\section{Conserved motif analysis}

Functional motifs or domains of PtHsp90 protein sequences were analyzed using PROSITE and the Conserved Domain database. MEME (http://meme.sdsc.edu) [22] was used to identify motifs in candidate sequences. MEME was run locally with the following parameters: number of repetitions = any, maximum number of motifs $=20$, and optimum motif width $=30$ to 70 residues.

\section{Transient expression and imaging}

Transient expression in Nicotiana benthamiana lower leaf epidermal cells was performed as described by Zheng et al. [45] with slight modifications. Plants were cultivated under short-day conditions $(8 \mathrm{~h}$ light/16 h dark). When the agrobacterium culture reached the stationary growth phase at $28^{\circ} \mathrm{C}$ with agitation, cells were pelleted and resuspended in infiltration buffer $(100 \mu \mathrm{M}$ acetosyringone in $10 \mathrm{mM} \mathrm{MgCl}_{2}$ ).

\section{Publicly available microarray data analyses}

For abiotic and hormonal treatments, Affymetrix microarray data available in the NCBI GEO database under the series accession numbers GSE26199 (heat stress), GSE17230 (drought stress) and GSE16786 were analyzed $[39,46,47]$. GSE16786 is composed of the following five subsets: GSE14893 (nitrogen limitation, genotype 1979), GSE14515 (nitrogen limitation, genotype 3200), GSE16783 (1 week after leaf wounding), GSE16785 (90 h after leaf wounding), and GSE16773 (methyl jasmonateelicited suspension cell cultures). The Affymetrix CEL files representing different abiotic and hormonal treatments were downloaded from the GEO database and preprocessed using GeneSpring GX (V11.5) software (Agilent Technologies). The data were normalized using the GCRMA algorithm and then log transformed. The averages were calculated. After normalization and log transformation of data for all of the Populus genes presented on the chip, the log signal intensity values for Populus probe IDs corresponding to the $H s p 90$ gene models (v1.1) were extracted as a subset for further analyses. Expression was shown as fold change in experimental treatment samples relative to control samples. Tab-delimited files for the average log signal intensity values were imported into Genesis (v1.75) to generate the heat maps [48].

Probe sets corresponding to PtHsp 90 genes were identified using the online Probe Match tool POParray (http:// aspendb.uga.edu/poparray). For probe sets matching 
several Populus Hsp 90 gene models, only these exhibiting consistently high hybridization signals across multiple samples were considered.

\section{Plant material and growth conditions}

Plant materials were collected from clonally propagated 1-year-old hybrid poplar $(P$. alba $\times P$. glandulosa $)$ clones (84K) grown in a growth chamber under long-day conditions (16 h light $/ 8 \mathrm{~h}$ dark) at $23-25^{\circ} \mathrm{C}$. Poplar saplings were subjected to heat treatment. Briefly, chamber was heated to $37^{\circ} \mathrm{C}$ for $3 \mathrm{~h}$ (pretreatment), returned to $23^{\circ} \mathrm{C}$ for $2 \mathrm{~h}$, heated to $45^{\circ} \mathrm{C}$ for $3 \mathrm{~h}$ (treatment), and then allowed to recover for $2 \mathrm{~h}$. Two biological replicates were performed. Leaves from three different plants were harvested at seven selected time points during heat stress treatment, frozen immediately in liquid nitrogen, and stored at $-80^{\circ} \mathrm{C}$ for further analysis.

\section{RNA isolation and semi-quantitative RT-PCR}

Total RNA was extracted using the RNeasy Plant Mini Kit (Qiagen) with on-column treatment with RNase-free DNase I (Qiagen) to remove any contamination of genomic DNA according to the manufacturer's instructions. RNA integrity was verified by $2 \%$ agar gel electrophoresis. First-strand cDNA synthesis was carried out with approximately $1 \mu \mathrm{g}$ RNA using the SuperScript III reverse transcription kit (Invitrogen) and random primers according to the manufacturer's procedure. Primers with melting temperatures of $58-60^{\circ} \mathrm{C}$, lengths of $20-27 \mathrm{bp}$, and amplicon lengths of 160-260 bp were designed using Primer3 software (http://frodo.wi.mit.edu/primer3/input. $\mathrm{htm})$. All primer sequences are listed in Additional file 13.

Real-time PCR was conducted on 7500 Real Time PCR System (Applied Biosystems, CA, USA) using SYBR Premix Ex Taq ${ }^{\text {TM }}$ Kit (TaKaRa, Tokyo, Japan). Reactions were prepared in a total volume of $20 \mu \mathrm{l}$ containing: $10 \mu \mathrm{l}$ of $2 \times$ SYBR Premix, $2 \mu \mathrm{l}$ of cDNA template, $0.4 \mu \mathrm{l}$ of each specific primer to a final concentration of $200 \mathrm{nM}$. The reactions were performed as the following conditions: initial denaturation step of $95^{\circ} \mathrm{C}$ for $30 \mathrm{~s}$ followed by two-step thermal cycling profile of denaturation at $95^{\circ} \mathrm{C}$ for $10 \mathrm{~s}$, and combined primer annealing/extension at $60^{\circ} \mathrm{C}$ for 34 $\mathrm{s}$ for 40 cycles. Negative PCR control without templates was performed for each primer pair. To verify the specificity of each primer pair, a melting curve analysis was performed ranging from $60^{\circ} \mathrm{C}$ to $95^{\circ} \mathrm{C}$ with temperature increasing steps of $0.06^{\circ} \mathrm{C} / \mathrm{s}$ ( 5 acquisitions per ${ }^{\circ} \mathrm{C}$ ) at the end of each run. The final threshold cycle $(\mathrm{Ct})$ values were the mean of eight values including two biological replicates for each treatment and four technical replicates. The PtActin gene was used as an internal control.

\section{Additional files}

Additional file 1: Conserved domains of Hsp90 proteins in
Arabidopsis, Populus, and rice. The major domains were identified
using Pfam (http://pfam.sanger.ac.uk/). A multiple alignment of Hsp90
proteins from Arabidopsis (At), Populus (Pt), and rice (Os) was performed
using Clustal X2.1, and a phylogenetic tree was constructed using MEGA
4.0 by the neighbor-joining (NJ) method with 1000 bootstrap replicates.

Additional file 2: List of all Hsp90 gene sequences identified in Populus and rice. The list comprises seven Arabidopsis Hsp90 sequences and Hsp90 sequences identified from Populus and rice in this study. Amino acid sequences were deduced from their corresponding coding sequences, and genomic DNA sequences were obtained from Phytozome (http://www. phytozome.net/poplar, release 2.1).

Additional file 3: List of Hsp90 protein sequences identified from eight plant species examined in this study.

Additional file 4: Distance and percentage of identity among Arabidopsis, Populus, and rice Hsp90 proteins. Amino acid identity among Populus, Arabidopsis, and rice Hsp90 proteins was analyzed in a pairwise fashion.

Additional file 5: Sequence logos for the conserved motifs of Hsp90 proteins in Arabidopsis, Populus, and rice. Conserved motifs and sequence logos were generated using the MEME search tool. Numbers on the horizontal axis represent sequence positions in the motifs and the vertical axis represents the information content in bits.

Additional file 6: Phylogenetic relationships of Hsp90 conserved motif sequences in Arabidopsis, Populus, and rice. A multiple alignment of Hsp90 proteins from A. thaliana (At), P. trichocarpa (Pt) and O. sativa (Os) was performed using Clustal X2.1, and a phylogenetic tree was constructed using conserved $\mathrm{Hsp} 90$ motif sequences by the maximum likelihood method with 1000 bootstrap replicates.

Additional file 7: Chromosomal locations of PtHsp90 genes. The schematic diagram shows the $10 \mathrm{Hsp} 90$ genes mapped to nine chromosomes. Homologous blocks derived from segmental duplication are indicated using the same colors. The diagram of genome-wide chromosome organization resulting from genome duplication events in Populus is adapted from Tuskan et al. [17].

Additional file 8: Chromosomal locations of Arabidopsis Hsp90 genes. The lines join the segmental duplicated homologous blocks. Additional file 9: Chromosomal locations of rice Hsp90 genes. The lines join the segmental duplicated homologous blocks that are indicated using the same colors.

Additional file 10: Gene duplication relationships in the Hsp90 gene family in Populus trichocarpa and Oryza sativa. Paralogous gene pairs generated by gene duplication within the Hsp90 family of $P$. trichocarpa $(A)$ and $O$. sativa $(B)$ were analyzed using the Plant Genome Duplication Database (http://chibba.agtec.uga.edu/duplication/). Each query gene displays only $\pm 500 \mathrm{~kb}$ regions. Gene lines connect gene pairs. Blue lines represent the other anchor gene pairs in the region, and the red line represents the query locus.

Additional file 11: RPKM of PtHsp90 genes in vegetative tissues obtained from RNA-seq data.

Additional file 12: Expression analysis of selected PtHsp90 genes under heat stress. A. Conditions of heat stress. Seedlings were heated to $37^{\circ} \mathrm{C}$ for $3 \mathrm{~h}$ (pretreatment), returned to $23^{\circ} \mathrm{C}$ for $2 \mathrm{~h}$, heated to $45^{\circ} \mathrm{C}$ for $3 \mathrm{~h}$ (treatment), and then allowed to recover for 2 h. 1, control; 2 , $30 \mathrm{~min}$ after pretreatment at $37^{\circ} \mathrm{C} ; 3,2 \mathrm{~h}$ after pretreatment at $37^{\circ} \mathrm{C} ; 4$, $1 \mathrm{~h}$ after recovery at $23^{\circ} \mathrm{C} ; 5,30$ min after treatment at $45^{\circ} \mathrm{C} ; 6,2 \mathrm{~h}$ after treatment at $45^{\circ} \mathrm{C} ; 7,2 \mathrm{~h}$ after recovery at $23^{\circ} \mathrm{C}$. B. Analysis of expression profiles of PtHsp90s in response to heat stress in Populus leaves by semiquantitative RT-PCR. The constitutively expressed PtActin was used as an internal control. Three independent experiments were performed under identical conditions. C-F. The relative mRNA abundance of four selected PtHsp90 genes was normalized with respect to reference gene PtActin under heat stress using qRT-PCR. Three biological replicates each with 
four technique replicates were performed and bars represent standard deviations (SD) of the replicates.

\section{Additional file 13: Primers used in RT-PCR analysis of the 10} PtHsp90 genes.

\section{Competing interest}

The authors declare that they have no competing interests.

\section{Authors' contributions}

JZ carried out all the analysis and interpreted the results. JL, LZ and BL helped in Populus materials collection and total RNA extraction. ML and JC conceived the project, supervised the analysis and critically revised the manuscript. All authors read and approved the final manuscript.

\section{Acknowledgements}

This work was supported by the National Basic Research Program of China [2012CB114500] and the National Natural Science Foundation of China [31030018] to ML, and the National High Technology Research and Development Program of China [2013AA102702] to JC.

Received: 25 January 2013 Accepted: 30 July 2013

Published: 5 August 2013

\section{References}

1. Wang W, Vinocur B, Shoseyov O, Altman A: Role of plant heat-shock proteins and molecular chaperones in the abiotic stress response. Trends Plant Sci 2004, 9(5):244-252.

2. Gupta SC, Sharma A, Mishra M, Mishra RK, Chowdhuri DK: Heat shock proteins in toxicology: how close and how far? Life Sci 2010 86(11-12):377-384.

3. Hu W, Hu G, Han B: Genome-wide survey and expression profiling of heat shock proteins and heat shock factors revealed overlapped and stress specific response under abiotic stresses in rice. Plant Sci 2009 176(4):583-590.

4. Krishna P, Gloor G: The Hsp90 family of proteins in Arabidopsis thaliana. Cell Stress Chaperone 2001, 6(3):238-246.

5. Young JC, Moarefi I, Hartl FU: Hsp90: a specialized but essential proteinfolding tool. J Cell Biol 2001, 154(2):267-273.

6. Richter K, Buchner J: Hsp90: chaperoning signal transduction. J Cell Physiol 2001, 188(3):281-290.

7. Milioni D, Hatzopoulos P: Genomic organization of hsp90 gene family in Arabidopsis. Plant Mol Biol 1997, 35(6):955-961.

8. Liu Y, Burch-Smith T, Schiff M, Feng S, Dinesh-Kumar SP: Molecular chaperone Hsp90 associates with resistance protein $\mathrm{N}$ and its signaling proteins SGT1 and Rar1 to modulate an innate immune response in plants. J Biol Chem 2004, 279(3):2101-2108.

9. Hubert DA, Tornero P, Belkhadir Y, Krishna P, Takahashi A, Shirasu K, Dangl JL: Cytosolic HSP90 associates with and modulates the Arabidopsis RPM1 disease resistance protein. EMBO J 2003, 22(21):5679-5689.

10. Imai J, Maruya M, Yashiroda H, Yahara I, Tanaka K: The molecular chaperone Hsp90 plays a role in the assembly and maintenance of the 26S proteasome. EMBO J 2003, 22(14):3557-3567.

11. Zhang Z, Quick MK, Kanelakis KC, Gijzen M, Krishna P: Characterization of a plant homolog of hop, a cochaperone of hsp90. Plant Physiol 2003, 131(2):525-535.

12. Rutherford SL, Lindquist S: Hsp90 as a capacitor for morphological evolution. Nature 1998, 396(6709):336-342.

13. Cao D, Froehlich JE, Zhang H, Cheng CL: The chlorate-resistant and photomorphogenesis-defective mutant cr88 encodes a chloroplasttargeted HSP90. Plant J 2003, 33(1):107-118.

14. Ishiguro S, Watanabe $Y$, Ito N, Nonaka H, Takeda N, Sakai T, Kanaya H, Okada K: SHEPHERD is the Arabidopsis GRP94 responsible for the formation of functional CLAVATA proteins. EMBO J 2002, 21(5):898-908.

15. Prassinos C, Haralampidis K, Milioni D, Samakovli D, Krambis K, Hatzopoulos P: Complexity of Hsp90 in organelle targeting. Plant Mol Biol 2008, 67(4):323-334.

16. Song H, Zhao R, Fan P, Wang X, Chen X, Li Y: Overexpression of AtHsp90.2, AtHsp90.5 and AtHsp90.7 in Arabidopsis thaliana enhances plant sensitivity to salt and drought stresses. Planta 2009, 229(4):955-964.

17. Tuskan GA, Difazio S, Jansson S, Bohlmann J, Grigoriev I, Hellsten U, Putnam N, Ralph S, Rombauts S, Salamov A, et al: The genome of black cottonwood, Populus trichocarpa (Torr. \& Gray). Science 2006, 313(5793):1596-1604.

18. Marchler-Bauer A, Lu S, Anderson JB, Chitsaz F, Derbyshire MK, DeWeeseScott C, Fong JH, Geer LY, Geer RC, Gonzales NR, et al: CDD: a conserved domain database for the functional annotation of proteins. Nucleic Acids Res 2011, 39:D225-229.

19. Marchler-Bauer A, Anderson JB, Chitsaz F, Derbyshire MK, DeWeese-Scott C, Fong JH, Geer LY, Geer RC, Gonzales NR, Gwadz M, et al: CDD: specific functional annotation with the conserved domain database. Nucleic Acids Res 2009, 37:D205-210

20. Dutta R, Inouye M: GHKL, An emergent ATPase/kinase superfamily. Trends Biochem Sci 2000, 25(1):24-28.

21. Hardison RC: A brief history of hemoglobins: plant, animal, protist, and bacteria. Proc Natl Acad Sci U S A 1996, 93(12):5675-5679.

22. Bailey TL, Williams N, Misleh C, Li WW: MEME: discovering and analyzing DNA and protein sequence motifs. Nucleic Acids Res 2006, 34:W369-373.

23. Obermann WM, Sondermann $H$, Russo AA, Pavletich NP, Hartl FU: In vivo function of Hsp90 is dependent on ATP binding and ATP hydrolysis. J Cell Biol 1998, 143(4):901-910.

24. Panaretou B, Prodromou C, Roe SM, O'Brien R, Ladbury JE, Piper PW, Pearl $\mathrm{LH}$ : ATP binding and hydrolysis are essential to the function of the Hsp90 molecular chaperone in vivo. EMBO J 1998, 17(16):4829-4836.

25. Kent WJ, Baertsch R, Hinrichs A, Miller W, Haussler D: Evolution's cauldron: duplication, deletion, and rearrangement in the mouse and human genomes. Proc Natl Acad Sci U S A 2003, 100(20):11484-11489.

26. Mehan MR, Freimer NB, Ophoff RA: A genome-wide survey of segmental duplications that mediate common human genetic variation of chromosomal architecture. Hum Genomics 2004, 1(5):335-344.

27. Blanc G, Hokamp K, Wolfe KH: A recent polyploidy superimposed on older large-scale duplications in the Arabidopsis genome. Genome Res 2003, 13(2):137-144

28. Guyot R, Keller B: Ancestral genome duplication in rice. Genome 2004, 47(3):610-614

29. Yoshida T, Ohama N, Nakajima J, Kidokoro S, Mizoi J, Nakashima K, Maruyama K, Kim J-M, Seki M, Todaka D, et al: Arabidopsis HsfA1 transcription factors function as the main positive regulators in heat shock-responsive gene expression. Mol Genet Genomics 2011, 286(5-6):321-332

30. Haseloff J, Siemering KR, Prasher DC, Hodge S: Removal of a cryptic intron and subcellular localization of green fluorescent protein are required to mark transgenic Arabidopsis plants brightly. Proc Natl Acad Sci USA 1997, 94(6):2122-2127.

31. Lin $Y$, Cheng $C L$ : A chlorate-resistant mutant defective in the regulation of nitrate reductase gene expression in Arabidopsis defines a new $\mathrm{HY}$ locus. Plant Cell 1997, 9(1):21-35.

32. Cao D, Lin Y, Cheng CL: Genetic interactions between the chlorateresistant mutant $\mathrm{cr} 88$ and the photomorphogenic mutants cop 1 and hy5. Plant Cell 2000, 12(2):199-210.

33. Felts SJ, Owen BA, Nguyen P, Trepel J, Donner DB, Toft DO: The hsp90related protein TRAP1 is a mitochondrial protein with distinct functional properties. J Biol Chem 2000, 275(5):3305-3312.

34. Gesualdi NM, Chirico G, Pirozzi G, Costantino E, Landriscina M, Esposito F: Tumor necrosis factor-associated protein 1 (TRAP-1) protects cells from oxidative stress and apoptosis. Stress 2007, 10(4):342-350.

35. Takabatake R, Ando Y, Seo S, Katou S, Tsuda S, Ohashi Y, Mitsuhara I: MAP kinases function downstream of HSP90 and upstream of mitochondria in TMV resistance gene $\mathrm{N}$-mediated hypersensitive cell death. Plant Cell Physiol 2007, 48(3):498-510.

36. Fletcher JC: Shoot and floral meristem maintenance in Arabidopsis. Annu Rev Plant Biol 2002, 53(1):45-66.

37. Sangster TA, Queitsch C: The HSP90 chaperone complex, an emerging force in plant development and phenotypic plasticity. Curr Opin Plant Biol 2005, 8(1):86-92.

38. Barrett T, Edgar R: Gene expression omnibus: microarray data storage, submission, retrieval, and analysis. Methods Enzymol 2006, 411:352-369.

39. Weston DJ, Karve AA, Gunter LE, Jawdy SS, Yang X, Allen SM, Wullschleger SD: Comparative physiology and transcriptional networks underlying the heat shock response in Populus trichocarpa, Arabidopsis thaliana and Glycine max. Plant Cell Environ 2011, 34(9):1488-1506.

40. Lescot M, Déhais $P$, Thijs $G$, Marchal $K$, Moreau $Y$, Van de Peer $Y$, Rouzé $P$, Rombauts S: PlantCARE, a database of plant cis-acting regulatory 
elements and a portal to tools for in silico analysis of promoter sequences. Nucleic Acids Res 2002, 30(1):325-327.

41. Larkin MA, Blackshields G, Brown NP, Chenna R, McGettigan PA, McWilliam H, Valentin F, Wallace IM, Wilm A, Lopez R, et al: Clustal W and Clustal X version 2.0. Bioinformatics 2007, 23(21):2947-2948.

42. Guindon S, Gascuel O: A simple, fast, and accurate algorithm to estimate large phylogenies by maximum likelihood. Syst Biol 2003, 52(5):696-704.

43. Jones DT, Taylor WR, Thornton JM: The rapid generation of mutation data matrices from protein sequences. Comput Appl Biosci 1992, 8(3):275-282.

44. Guo AY, Zhu QH, Chen X, Luo JC: GSDS: a gene structure display server. Yi chuan 2007, 29(8):1023-1026.

45. Zheng H, Camacho L, Wee E, Batoko H, Legen J, Leaver CJ, Malho R, Hussey PJ, Moore I: A Rab-E GTPase mutant acts downstream of the Rab-D subclass in biosynthetic membrane traffic to the plasma membrane in tobacco leaf epidermis. Plant Cell 2005, 17(7):2020-2036.

46. Cohen D, Bogeat-Triboulot MB, Tisserant E, Balzergue S, Martin-Magniette ML, Lelandais G, Ningre N, Renou JP, Tamby JP, Le Thiec D, et al: Comparative transcriptomics of drought responses in Populus: a metaanalysis of genome-wide expression profiling in mature leaves and root apices across two genotypes. BMC Genomics 2010, 11(1):630.

47. Yuan Y, Chung JD, Fu X, Johnson VE, Ranjan P, Booth SL, Harding SA, Tsai $\mathrm{CJ}$ : Alternative splicing and gene duplication differentially shaped the regulation of isochorismate synthase in Populus and Arabidopsis. Proc Natl Acad Sci U S A 2009, 106(51):22020-22025.

48. Sturn A, Quackenbush J, Trajanoski Z: Genesis: cluster analysis of microarray data. Bioinformatics 2002, 18(1):207-208.

doi:10.1186/1471-2164-14-532

Cite this article as: Zhang et al:: Genome-wide analysis of the Populus Hsp90 gene family reveals differential expression patterns, localization, and heat stress responses. BMC Genomics 2013 14:532.

\section{Submit your next manuscript to BioMed Central and take full advantage of:}

- Convenient online submission

- Thorough peer review

- No space constraints or color figure charges

- Immediate publication on acceptance

- Inclusion in PubMed, CAS, Scopus and Google Scholar

- Research which is freely available for redistribution 